\title{
3 Research Square

\section{Human Umbilical Cord-Blood Derived Mesenchymal Stem Cell Can Improve the Clinical Outcome and Joint Space Width after High Tibial Osteotomy}

\section{Dong Won Suh}

Barunsesang Hospital

\section{Seung Beom Han}

Korea University Medical Center

Woo Jin Yeo

Barunsesang Hospital

Kuhoang Cheong

Barunsesang Hospital

Sang-Yeon So

Barunsesang Hospital

Bong Soo Kyung ( $\square$ id-helios27@hanmail.net)

Barunsesang Hospital

\section{Research Article}

Keywords: knee, medial osteoarthritis, cartilage defect, umbilical cord-blood-derived mesenchymal stem cell, high tibial osteotomy

Posted Date: March 10th, 2021

DOl: https://doi.org/10.21203/rs.3.rs-257427/v1

License: (9) This work is licensed under a Creative Commons Attribution 4.0 International License.

Read Full License

Version of Record: A version of this preprint was published at The Knee on December 1st, 2021. See the published version at https://doi.org/10.1016/j.knee.2021.08.028. 


\section{Abstract \\ Purpose}

We compared the clinical and radiologic results of human umbilical cord-blood derived mesenchymal stem cells (hUCB-MSCs)cartilage regeneration procedures combined with HTO to those of microfracture with HTO.

\section{Methods}

From August 2017 to December 2018, HTO patients with International Cartilage Regeneration and Joint Preservation Society (ICRS) grade IV cartilage defects over $200 \mathrm{~mm}^{2}$ on medial femoral condyle (MFC) were enrolled. For comparison, all participants were divided into two groups: those who had undergone an hUCB-MSC induced cartilage regeneration procedure (group MSC) and those with microfractures only (group C, controls). Clinically, Hospital for Special Surgery (HSS), International Knee Documentation Committee (IKDC), and Lysholm scores were evaluated post-operatively at 18 months. Radiologically, mechanical axis (MA) and joint space width (JSW) were evaluated.

\section{Results}

A total of 100 knees were enrolled (43 in group MSC, 57 in group C). The IKDC score in group MSC (69) was better than that in group $C(62 ; P<0.05)$. The JSW increment in the MSC group $(0.6 \mathrm{~mm})$ was more than that in group $C(0.1 \mathrm{~mm} ; P<0.05)$. No patient developed nonunion, correction loss, or arthroplasty conversion.

\section{Conclusion}

hUCB-MSCs can improve clinical outcome and JSW better than microfracture only in HTO patients.

\section{Introduction}

High tibial osteotomy (HTO) is a well-established surgery for patients with medial osteoarthritis (OA) with varus alignment. Many studies have reported long-term survival to be over $80 \% .{ }^{1-6}$ Furthermore, a combined cartilage regeneration procedure such as microfracture (MFx) has an even better survival rate in the long-term. ${ }^{7-9}$ Failed cartilage regeneration is closely related to the early failure of HTO and the need for total replacement arthroplasty.

Various methods have been proposed for cartilage regeneration. MFx has been used widely; however, there are concerns with this procedure, such as fibrocartilage formation and its unsuitability for arthritic cartilage defects. ${ }^{10-12}$ Various augmentation methods, such as collagen substitutes, osteochondral 
grafts, and autologous chondrocyte implantation have been suggested, but the use of these is limited to elderly patients with $\mathrm{OA} .^{13,14}$ Since the development of cell therapy, various groups have used mesenchymal stem cells (MSCs) for cartilage regeneration, including autologous bone marrow aspirate concentrate (BMAC), autologous fat-derived stromal vascular fraction, autologous stem cells from fat or

synovial tissue, and human umbilical cord-blood derived mesenchymal stem cells (hUCB-MSCs). ${ }^{15-20}$

Previous studies have shown good results for cartilage regeneration using MSCs in HTO patients. ${ }^{16-18}$ However, there is still concern about using autologous MSCs for cartilage regeneration in elderly patients. ${ }^{21-24}$ Because HTO is used commonly in patients who are elderly and suffer with advanced OA, another surgical option, such as allogenic MSC therapy, is needed. One option for cartilage regeneration in old age is allogenic hUCB-MSCs, an allogenic MSC therapy for cartilage regeneration that has been used for over 50 years and is known as a successful cartilage regeneration technique. ${ }^{25}$

However, there are limited studies about hUCB-MSCs. In the current study, we evaluated the clinical and radiological outcomes of patients who had undergone hUCB-MSC treatment combined with HTO compared with those of patients who had undergone MFx combined with HTO. The hypothesis was that hUCB-MSC-treated patients would have better clinical and radiological outcomes than MFx in HTO patients.

\section{Materials And Methods}

The protocol and design of the current study were reviewed and approved by the Ethics Committee of the Public Institutional Review Board Designated by Ministry of Health and Welfare (IRB approval 2020-2698001).

Medical records and radiological results of HTO patients were retrospectively reviewed to compare them based on the use of hUCB-MSC treatment and MFx only.

The written informed consent were obtained for all participants before surgery, and all relevant guidelines were followed for the study

Inclusion criteria of the study were

1. International Cartilage Regeneration and Joint Preservation Society (ICRS) grade IV cartilage defect of medial femoral condyle (MFC) over $200 \mathrm{~mm}^{2}$

2. Medial opening-wedge HTO fixed by locking plate with metal wedge (OhtoFix type I plate; Ohtomedical Co. Ltd., Goyang, Korea)

3. A minimum follow-up of 18 months after surgery

4. All surgery performed in a single institution

\section{Surgical procedures and postoperative rehabilitation protocol}


Preoperatively, the correction amount was measured using Miniaci's method, targeting the lateral tibial spine at the standing anteroposterior (AP) view of the whole lower extremity using the PiView STAR (INFINITT, Seoul, South Korea), a type of picture archiving and communication system (PACS).

Under spinal anesthesia, standard anteromedial and anterolateral portals were made. Arthroscopic examination was performed, including synovectomy, debridement, partial meniscectomy, and/or meniscal repair. For cartilage defects of MFC, radial debridement was performed at the subchondral bone level. Then, an anteromedial portal was extended about $2 \mathrm{~cm}$. Using a $4 \mathrm{~mm}$-diameter reamer, multiple 4 $\mathrm{mm}$-deep holes were made in subchondral bone in cartilage defects. After saline drainage, off-the-shelf hUCB-MSC product, Cartistem (MEDIPOST, Seongnam, South Korea), was applied. This product consists of $1.5 \mathrm{~mL}$ hUCB-MSCs $\left(7.5 \times 10^{6} \mathrm{cells} / \mathrm{vial}\right)$ and $4 \%$ hyaluronic acid hydrogel, and it was approved by the Korea Food and Drug Administration in 2012 and is used for cartilage regeneration ${ }^{15,19,20,25}$ (Fig. 1). After arthroscopic examination and hUCB-MSC implantation, medial opening-wedge HTO was performed conventionally using an anatomical locking plate with metal wedge (Ohtofix type I). ${ }^{26}$

Postoperative rehabilitation protocol, early weight-bearing, was the same in both groups. Patients started continuous passive motion exercise and were given a hinged knee-brace postoperative day 2 after drainage removal. At postoperative day 7 , partial weight bearing was permitted with crutch. Tolerable fullweight bearing was allowed at postoperative 4 weeks. Patients wore the hinged knee-brace for 8 weeks. Plate removal was recommended after 18 months, after confirming union by radiographs.

\section{Evaluation and statistical analysis}

At postoperative day 2, all patients had magnetic resonance imaging (MRI) to determine if any of them had lateral hinge fracture or other problems. Follow-up in the outpatient department was recommended at $2,6,12$, and 18 months after the operation. At each follow-up, radiographs, including the Rosenberg view, lateral view, and standing AP view of the whole lower extremity were taken, and the joint space width (JSW) in Rosenberg ${ }^{27}$ and the mechanical femorotibial angle (MA) in the standing AP view of the whole lower extremity were measured in the previous PACS viewer. The minimum differences that the software could detect were $0.1^{\circ}$ in angle and $0.1 \mathrm{~mm}$ in length. At postoperative 18 months, clinical outcome was checked by the Hospital for Special Surgery (HSS), Lysholm, and International Knee Documentation Committee (IKDC) subjective scores.

Post-hoc power analysis for effect size, using a two-sided hypothesis test to obtain a power of 0.8 and a significance level of 0.05 , reported that 41 knees for each group were considered adequate for detection of significant differences in IKDC subjective score between the two groups.

Statistically, the pre- and postoperative MA (+, mean varus; -, mean valgus) and JSW, difference in MA, JSW increment, size of wedge, size of cartilage defect, the postoperative HSS, Lysholm, and IKDC subjective scores were compared by Student's $t$ test. The Statistical Package for the Social Sciences (SPSS) 12.0 (SPSS Science, Inc., Chicago, IL, US) was used for all statistical analyses, and $P<0.05$ was considered to be statistically significant. 


\section{Results}

From August 2017 to December 2018, a total of 100 knee joints were enrolled in the current study; 43 knees were implanted with hUCB-MSCs for cartilage regeneration (group MSC), and the other 57 knees underwent MFx only (group C). The patients' demographics are shown in Table 1; there were no significant differences between the two groups.

Intra-operatively, mean cartilage defects were $442 \pm 210 \mathrm{~mm}^{2}$ in the MSC group and $401 \pm 162 \mathrm{~mm}^{2}$ in group $\mathrm{C}$, and there was no significant difference between the two groups. Mean sizes of the wedge were $10.0 \pm 2.0 \mathrm{~mm}$ and $10.3 \pm 1.9 \mathrm{~mm}$ in the MSC group and group C, respectively, and there was also no significant difference between the two groups (Table 1).

Radiologically, mean pre-MA, post-MA, and the difference in MA were $6.7 \pm 2.2,-1.5 \pm 1.4$, and $-8.1 \pm 2.3$ degrees in the MSC group and 7.3 $\pm 2.3,-1.3 \pm 1.7$, and $-8.7 \pm 2.7$ degrees in group $C$. In the MSC group, mean pre-JSW and post-JSW were $3.0 \pm 1.7 \mathrm{~mm}$ and $3.6 \pm 1.5 \mathrm{~mm}$, and the JSW increment was $0.6 \pm 0.9$ $\mathrm{mm}$. In group C, mean pre-JSW and post-JSW were $3.7 \pm 1.7 \mathrm{~mm}$ and $3.8 \pm 1.8 \mathrm{~mm}$, and the increment was $0.1 \pm 1.3 \mathrm{~mm}$. There was a statistically significant difference in the JSW increment between the two groups (Table 2). There were no severe complications, such as nonunion, correction loss, or arthroplasty conversion, in either group.

Clinically, mean postoperative HSS, Lysholm, and IKDC subjective scores were $92.5 \pm 3.9,69.0 \pm 7.7$, and $84.0 \pm 11.2$ in the MSC group and $90.2 \pm 6.2,62.1 \pm 12.9$, and $79.2 \pm 13.0$ in group C. There was a statistically significant difference in the IKDC subjective score between the two groups (Table 2). A 60year-old male patient in the MSC group showed good cartilage regeneration in second-look arthroscopy, and he had a JSW increment of $1.3 \mathrm{~mm}$ and good clinical results: 91 in HSS, 80 in Lysholm, and 71 in IKDC subjective score (Fig. 2).

\section{Discussion}

The main purpose of the current study was to evaluate the effect of hUCB-MSC treatment in HTO patients by comparing the clinical and radiological outcomes between two groups. The results showed better clinical and radiological outcomes in the MSC group at postoperative 18 months. This is the first study to compare hUCB-MSC treatment and MFx in HTO patients and to show that hUCB-MSC had better clinical results and JSW increments in early follow-up.

HTO is a good surgical option for advanced OA with varus alignment. Previous HTO studies about patients with advanced OA reported good results in the long-term. ${ }^{3,5,28}$ Schuster et al reported on the longterm survival of HTO in patients with severe OA. They reported $80 \%$ survival at 10 -year follow-up with MFx chondroplasty. ${ }^{5}$ The current study evaluated patients with advanced ICRS grade IV cartilage defects over $200 \mathrm{~mm}^{2}$ on MFC, and both groups reported good clinical results at 18 month follow-up. These results show the effectiveness of HTO for patients with advanced OA in the knees. 
For patients with advanced $O A$, cartilage regeneration can result in better clinical outcome. ${ }^{16,18,29,30}$ Koh et al reported that autologous fat-derived stromal vascular fraction had better cartilage regeneration and clinical results than MFx only in HTO patients. ${ }^{30} \mathrm{Jin}$ et al reported autologous BMAC in HTO and better cartilage regeneration in the BMAC group than in the MFx group. ${ }^{16} \mathrm{Kim}$ et al studied collagen augmentation in HTO compared with MFx alone, and their results were similar to previous studies. ${ }^{29}$ The current study agreed with these studies: allogenic hUCB-MSC augmentation resulted in better IKDC scores and more JSW increments than MFx only in HTO patients with cartilage defects over $200 \mathrm{~mm}^{2}$. These results showed that cartilage regeneration procedures combined with HTO result in better cartilage regeneration and better clinical outcome.

However, there are concerns about using autologous cell cartilage augmentation procedures in older patients. Autologous chondrocyte implantation is not suitable in patients over $45 .{ }^{13,14}$ In a previous study about BMAC, cell counts and the differential potency of BMAC in elderly patients were lower than in younger patients, which suggests against using BMAC in elderly patients. ${ }^{24}$ Previous laboratory studies on MSC and age reported that elderly patients had lower cell counts and lower proliferation of MSCs derived from autologous bone marrow or adipose tissue. ${ }^{22,23}$ They suggested the necessity of allogenic MSCs in elderly patients, such as hUCB-MSCs. The current study was initiated to investigate the use of allogenic MSCs for cartilage regeneration in elderly patients with OA.

The hUCB-MSCs, allogenic stem cells from umbilical cord-blood, have enough growth factors and cytokines for regeneration, ${ }^{25}$ which can affect cartilage regeneration in patients over 50 . Park et al reported that hUCB-MSCs made more hyaline-like cartilage in patients over 50 than MFx. ${ }^{25}$ These findings suggest that hUCB-MSCs result in better cartilage regeneration than other cartilage augmentation procedures in HTO patients over 50 years old. Previous case series about HTO with hUCB-MSCs reported good clinical outcomes and cartilage regeneration. ${ }^{15,19,20}$ The current study agreed with these results, with good clinical outcomes and JSW increments in elderly patients.

The JSW in Rosenberg view is a relevant numeric value showing the cartilage regeneration and progression of OA. Previous studies used the JSW as the cartilage thickness and considered it the simplest value of OA progression. ${ }^{27,31,32}$ The current study also used the JSW as a radiological result to evaluate cartilage regeneration, because postoperative MRI and second-look arthroscopy were not applied routinely. In the current study, JSW increment and postoperative IKDC score were better in the MSC group than in the control group. Considering the relationship between cartilage regeneration and clinical outcome, these results show the hUCB-MSCs may reinforce cartilage regeneration in HTO patients with cartilage defects over $200 \mathrm{~mm}^{2}$.

There were limitations in the study. First, it was a small, not-randomized study. Second, the follow-up period was short. Third, there were no pre-operative clinical data. Fourth, there were no detailed data on cartilage regeneration, such as MRIs or second-look arthroscopy. However, the demographics and pre-op radiological data were not different in the two groups, and a statistically significant difference was found 
in postoperative 18-month IKDC scores and JSW differences. This showed the probability of effectiveness of the hUCB-MSCs. Considering that the previous studies about long-term results of HTO were related to cartilage regeneration, hUCB-MSC treatment combined with $\mathrm{HTO}$ is a good option for patients with advanced $O A$ who are over 50 years old.

\section{Conclusions}

hUCB-MSC treatment can improve clinical outcomes and JSW more than MFx only in HTO patients.

\section{References}

1 Akizuki, S., Shibakawa, A., Takizawa, T., Yamazaki, I. \& Horiuchi, H. The long-term outcome of high tibial osteotomy: a ten- to 20-year follow-up. The Journal of bone and joint surgery. British volume 90 , 592-596, doi:10.1302/0301-620x.90b5.20386 (2008).

2 Hui, C. et al. Long-term survival of high tibial osteotomy for medial compartment osteoarthritis of the knee. The American journal of sports medicine 39, 64-70, doi:10.1177/0363546510377445 (2011).

3 Ollivier, B., Berger, P., Depuydt, C. \& Vandenneucker, H. Good long-term survival and patient-reported outcomes after high tibial osteotomy for medial compartment osteoarthritis. Knee surgery, sports traumatology, arthroscopy : official journal of the ESSKA, doi:10.1007/s00167-020-06262-4 (2020).

4 Sasaki, E. et al. Long-term survival rate of closing wedge high tibial osteotomy with high valgus correction: a 15-year follow-up study. Knee surgery, sports traumatology, arthroscopy : official journal of the ESSKA, doi:10.1007/s00167-020-06128-9 (2020).

5 Schuster, P. et al. Ten-Year Results of Medial Open-Wedge High Tibial Osteotomy and Chondral Resurfacing in Severe Medial Osteoarthritis and Varus Malalignment. The American journal of sports medicine 46, 1362-1370, doi:10.1177/0363546518758016 (2018).

6 Spahn, G. et al. The impact of a high tibial valgus osteotomy and unicondylar medial arthroplasty on the treatment for knee osteoarthritis: a meta-analysis. Knee surgery, sports traumatology, arthroscopy: official journal of the ESSKA 21, 96-112, doi:10.1007/s00167-011-1751-2 (2013).

7 Harris, J. D., McNeilan, R., Siston, R. A. \& Flanigan, D. C. Survival and clinical outcome of isolated high tibial osteotomy and combined biological knee reconstruction. The Knee 20, 154-161, doi:10.1016/j.knee.2012.12.012 (2013).

8 Pascale, W., Luraghi, S., Perico, L. \& Pascale, V. Do microfractures improve high tibial osteotomy outcome? Orthopedics 34, e251-255, doi:10.3928/01477447-20110526-06 (2011).

9 Kahlenberg, C. A., Nwachukwu, B. U., Hamid, K. S., Steinhaus, M. E. \& Williams, R. J., 3rd. Analysis of Outcomes for High Tibial Osteotomies Performed With Cartilage Restoration Techniques. Arthroscopy: 
the journal of arthroscopic \& related surgery : official publication of the Arthroscopy Association of North America and the International Arthroscopy Association 33, 486-492, doi:10.1016/j.arthro.2016.08.010 (2017).

10 Goyal, D., Keyhani, S., Lee, E. H. \& Hui, J. H. Evidence-based status of microfracture technique: a systematic review of level I and II studies. Arthroscopy : the journal of arthroscopic \& related surgery : official publication of the Arthroscopy Association of North America and the International Arthroscopy Association 29, 1579-1588, doi:10.1016/j.arthro.2013.05.027 (2013).

11 Gobbi, A., Karnatzikos, G. \& Kumar, A. Long-term results after microfracture treatment for fullthickness knee chondral lesions in athletes. Knee surgery, sports traumatology, arthroscopy : official journal of the ESSKA 22, 1986-1996, doi:10.1007/s00167-013-2676-8 (2014).

12 Scotti, C. et al. Cartilage Repair in the Inflamed Joint: Considerations for Biological Augmentation Toward Tissue Regeneration. Tissue engineering. Part B, Reviews 22, 149-159, doi:10.1089/ten.TEB.2015.0297 (2016).

13 Knutsen, G. et al. Autologous chondrocyte implantation compared with microfracture in the knee. A randomized trial. The Journal of bone and joint surgery. American volume 86, 455-464, doi:10.2106/00004623-200403000-00001 (2004).

14 Rosenberger, R. E., Gomoll, A. H., Bryant, T. \& Minas, T. Repair of large chondral defects of the knee with autologous chondrocyte implantation in patients 45 years or older. The American journal of sports medicine 36, 2336-2344, doi:10.1177/0363546508322888 (2008).

15 Chung, Y. W., Yang, H. Y., Kang, S. J., Song, E. K. \& Seon, J. K. Allogeneic umbilical cord bloodderived mesenchymal stem cells combined with high tibial osteotomy: a retrospective study on safety and early results. International orthopaedics, doi:10.1007/s00264-020-04852-y (2020).

16 Jin, Q. H. et al. Bone marrow aspirate concentration provided better results in cartilage regeneration to microfracture in knee of osteoarthritic patients. Knee surgery, sports traumatology, arthroscopy : official journal of the ESSKA, doi:10.1007/s00167-020-06099-x (2020).

17 Kim, Y. S. et al. Implantation of mesenchymal stem cells in combination with allogenic cartilage improves cartilage regeneration and clinical outcomes in patients with concomitant high tibial osteotomy. Knee surgery, sports traumatology, arthroscopy : official journal of the ESSKA 28, 544-554, doi:10.1007/s00167-019-05729-3 (2020).

18 Kim, Y. S. \& Koh, Y. G. Comparative Matched-Pair Analysis of Open-Wedge High Tibial Osteotomy With Versus Without an Injection of Adipose-Derived Mesenchymal Stem Cells for Varus Knee Osteoarthritis: Clinical and Second-Look Arthroscopic Results. The American journal of sports medicine 46, 2669-2677, doi:10.1177/0363546518785973 (2018). 
19 Song, J. S., Hong, K. T., Kim, N. M., Park, H. S. \& Choi, N. H. Human umbilical cord blood-derived mesenchymal stem cell implantation for osteoarthritis of the knee. Archives of orthopaedic and trauma surgery 140, 503-509, doi:10.1007/s00402-020-03349-y (2020).

20 Song, J. S. et al. High tibial osteotomy with human umbilical cord blood-derived mesenchymal stem cells implantation for knee cartilage regeneration. World journal of stem cells 12, 514-526, doi:10.4252/wjsc.v12.i6.514 (2020).

21 Baker, N., Boyette, L. B. \& Tuan, R. S. Characterization of bone marrow-derived mesenchymal stem cells in aging. Bone 70, 37-47, doi:10.1016/j.bone.2014.10.014 (2015).

22 Choudhery, M. S., Badowski, M., Muise, A., Pierce, J. \& Harris, D. T. Donor age negatively impacts adipose tissue-derived mesenchymal stem cell expansion and differentiation. Journal of translational medicine 12, 8, doi:10.1186/1479-5876-12-8 (2014).

23 Wu, L. W. et al. Donor age negatively affects the immunoregulatory properties of both adipose and bone marrow derived mesenchymal stem cells. Transplant immunology 30, 122-127, doi:10.1016/j.trim.2014.03.001 (2014).

24 Payne, K. A., Didiano, D. M. \& Chu, C. R. Donor sex and age influence the chondrogenic potential of human femoral bone marrow stem cells. Osteoarthritis and cartilage 18, 705-713, doi:10.1016/j.joca.2010.01.011 (2010).

25 Park, Y. B., Ha, C. W., Lee, C. H., Yoon, Y. C. \& Park, Y. G. Cartilage Regeneration in Osteoarthritic Patients by a Composite of Allogeneic Umbilical Cord Blood-Derived Mesenchymal Stem Cells and Hyaluronate Hydrogel: Results from a Clinical Trial for Safety and Proof-of-Concept with 7 Years of Extended Follow-Up. Stem cells translational medicine 6, 613-621, doi:10.5966/sctm.2016-0157 (2017).

26 Suh, D. W., Kyung, B. S., Han, S. B., Cheong, K. \& Lee, W. H. Efficacy of Tranexamic Acid for Hemostasis in Patients Undergoing High Tibial Osteotomy. The journal of knee surgery 31, 50-55, doi:10.1055/s-0037-1600091 (2018).

27 Park, C. H., Bae, D. K., Kim, K. I., Lee, J. W. \& Song, S. J. Serial Changes in the Joint Space Width and Joint Line Convergence Angle After Closed-Wedge High Tibial Osteotomy. The American journal of sports medicine 45, 3254-3261, doi:10.1177/0363546517729153 (2017).

28 Pannell, W. C. et al. High Tibial Osteotomy Survivorship: A Population-Based Study. Orthopaedic journal of sports medicine 7, 2325967119890693, doi:10.1177/2325967119890693 (2019).

29 Kim, M. S., Koh, I. J., Choi, Y. J., Pak, K. H. \& In, Y. Collagen Augmentation Improves the Quality of Cartilage Repair After Microfracture in Patients Undergoing High Tibial Osteotomy: A Randomized Controlled Trial. The American journal of sports medicine 45, 1845-1855, doi:10.1177/0363546517691942 (2017). 
30 Koh, Y. G., Kwon, O. R., Kim, Y. S. \& Choi, Y. J. Comparative outcomes of open-wedge high tibial osteotomy with platelet-rich plasma alone or in combination with mesenchymal stem cell treatment: a prospective study. Arthroscopy : the journal of arthroscopic \& related surgery : official publication of the Arthroscopy Association of North America and the International Arthroscopy Association 30, 1453-1460, doi:10.1016/j.arthro.2014.05.036 (2014).

31 van der Woude, J. A. D. et al. Knee joint distraction compared with high tibial osteotomy: a randomized controlled trial. Knee surgery, sports traumatology, arthroscopy : official journal of the ESSKA 25, 876-886, doi:10.1007/s00167-016-4131-0 (2017).

32 Nha, K. W. et al. Radiological grading of osteoarthritis on Rosenberg view has a significant correlation with clinical outcomes after medial open-wedge high-tibial osteotomy. Knee surgery, sports traumatology, arthroscopy : official journal of the ESSKA 27, 2021-2029, doi:10.1007/s00167-018-5121-1 (2019).

\section{Tables}

Table 1. Patient demographics (range)

\begin{tabular}{lccl}
\hline & Group MSC & Group C & p value * \\
\hline N & 43 & 57 & - \\
Sex (M/F) & $15 / 28$ & $12 / 45$ & ns \\
Age (yrs old) & $56.5(48-65)$ & $58.6(48-66)$ & ns \\
Heights (cm) & $162(150-180)$ & $160(149-179)$ & ns \\
Weights (Kg) & $71(53-92)$ & $67(51-89)$ & ns \\
Body mass index (BMI) & $27.0(21-34)$ & $26.3(21-34)$ & ns \\
Mechanical axis (9) & $6.7(2.9-12.5)$ & $7.3(3.2-14.0)$ & ns \\
Joint space width, mm & $3.0(0-7.6)$ & $3.7(0-7.7)$ & ns \\
Defect on MFC, mm & $442(200-1200)$ & $401(200-800)$ & ns \\
Size of wedge, mm & $10.0(6-14)$ & $10.3(7-15)$ & ns \\
\hline
\end{tabular}

MFC, medial femoral condyle 
*Student's $t$ test

$P<0.05$, statistical significance

Table 2. Clinical and radiologic results ( \pm standard deviation)

\begin{tabular}{lllll}
\hline & & Group MSC & Group C & p value * \\
\hline Clinical outcome & Lysholm & $92.5 \pm 3.9$ & $90.2 \pm 6.2$ & ns \\
& IKDC & $69.0 \pm 7.7$ & $62.1 \pm 12.9$ & $\mathbf{0 . 0 0 2}$ \\
& HSS & $84.0 \pm 11.2$ & $79.2 \pm 13.0$ & ns \\
Radiological & Mechanical axis $\left({ }^{\circ}\right)$ & $-1.5 \pm 1.4$ & $-1.3 \pm 1.7$ & ns \\
outcome & MA diff. $\left({ }^{\circ}\right)$ & $-8.1 \pm 2.3$ & $-8.7 \pm 2.7$ & ns \\
& Joint Space Width $(\mathrm{mm})$ & $3.6 \pm 1.5$ & $3.8 \pm 1.8$ & ns \\
& JSW increment $(\mathrm{mm})$ & $0.6 \pm 0.9$ & $0.1 \pm 1.3$ & $\mathbf{0 . 0 3 6}$ \\
\hline
\end{tabular}

IKDC, International Knee Documentation Committee; HSS, Hospital for Special Surgery; MA, mechanical axis; JSW, joint space width

*Student's $t$ test

$P<0.05$, statistical significance

\section{Figures}




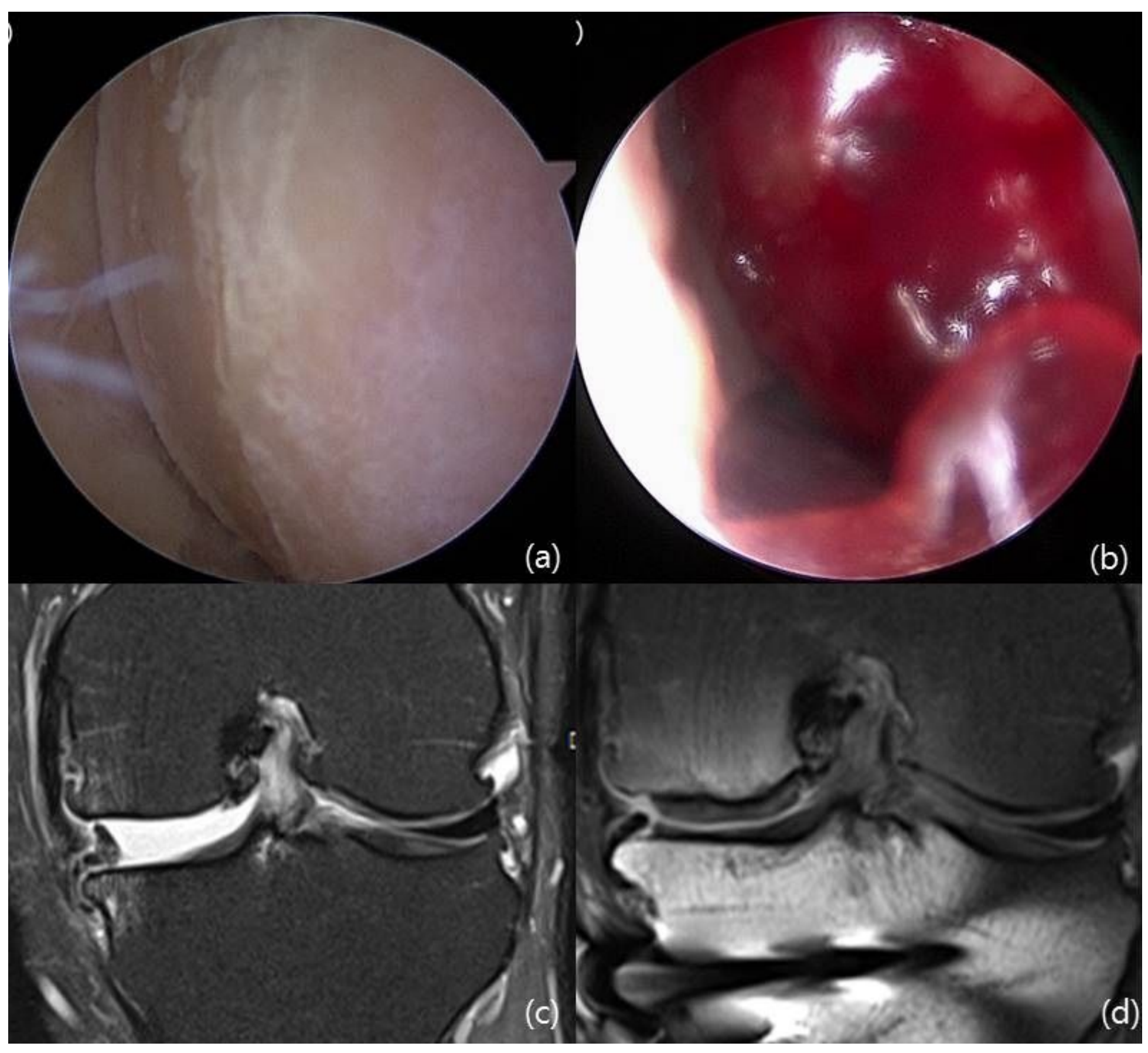

\section{Figure 1}

Cartilage augmentation procedure using human umbilical cord-blood derived mesenchymal stem cells (hUCB-MSCs) with hyaluronic acid hydrogel. (a) On chondral defect of medial femoral condyle (MFC), radical debridement and multiple $4 \mathrm{~mm}$ holes $4 \mathrm{~mm}$ deep were drilled on subchondral bone. (b) After fluid drainage, a mixture of hUCB-MSCs with hyaluronic acid hydrogel (Cartistem; MEDIPOST, Seongnam, Korea) was applied on the defect site. (c,d) A cartilage defect of MFC was filled with regenerated cartilage, as shown with magnetic resonance imaging (MRI) after 18 months. 


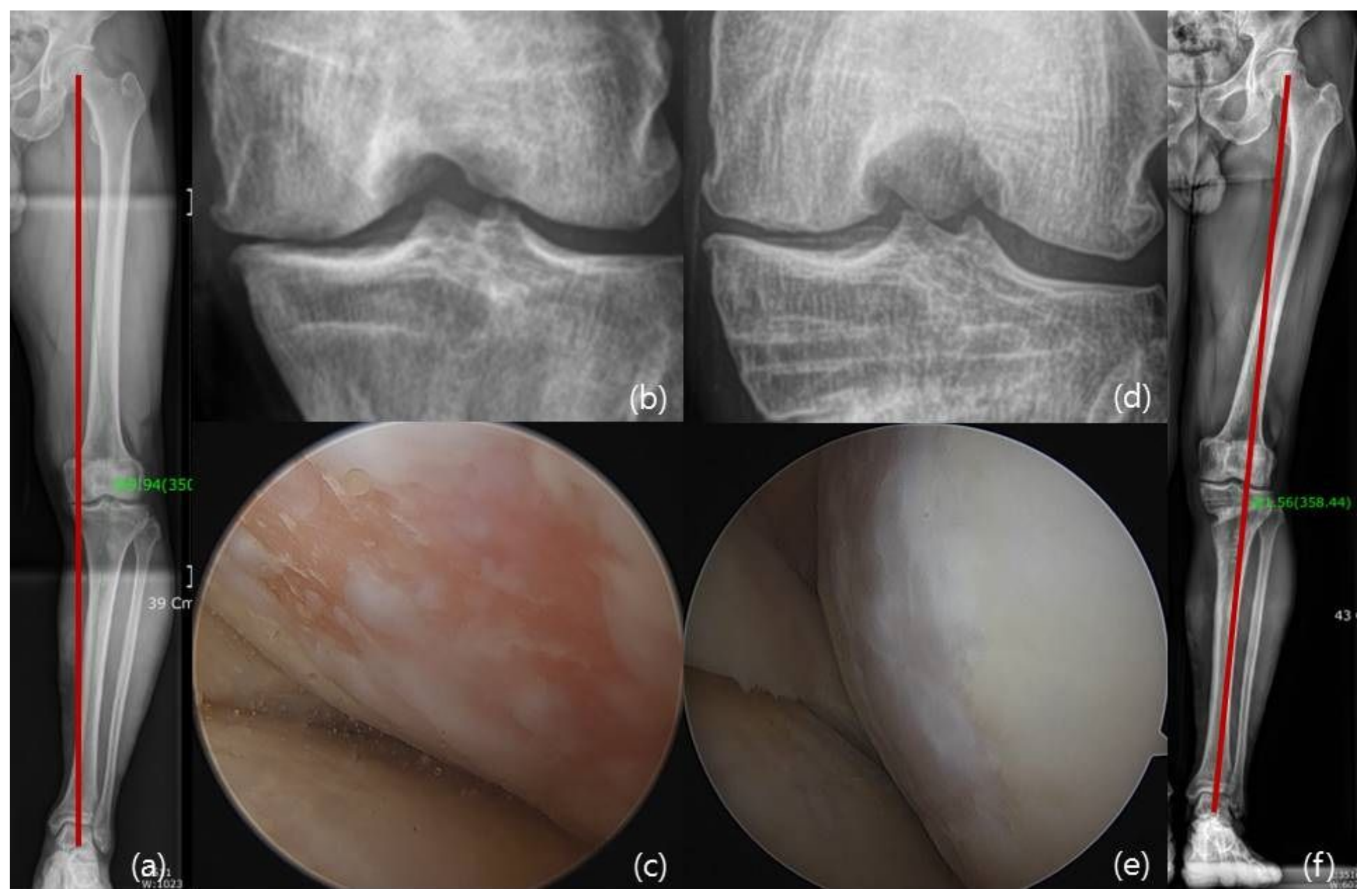

\section{Figure 2}

Mesenchymal stem cells (MSCs). (a,b) A male 60-year-old patient showed varus alignment, and his joint space width (JSW) was narrowed to $1.6 \mathrm{~mm}$ preoperatively. (c) Intra-operatively, a $500 \mathrm{~mm} 2$ cartilage defect was identified on medial femoral condyle (MFC) and human umbilical cord-blood derived mesenchymal stem cell (hUCB-MSC) application combined with HTO was performed. After 18 months (df), slightly valgus alignment was maintained with the JSW increased to $2.9 \mathrm{~mm}$. On second-look arthroscopy, cartilage was well regenerated. 